\title{
THE SOLUTION OF THE THIRD PROBLEM FOR THE LAPLACE EQUATION ON PLANAR DOMAINS WITH SMOOTH BOUNDARY AND INSIDE CRACKS AND MODIFIED JUMP CONDITIONS ON CRACKS
}

\author{
DAGMAR MEDKOVÁ
}

Received 23 November 2005; Revised 13 March 2006; Accepted 4 April 2006

This paper studies the third problem for the Laplace equation on a bounded planar domain with inside cracks. The third condition $\partial u / \partial n+h u=f$ is given on the boundary of the domain. The skip of the function $u_{+}-u_{-}=g$ and the modified skip of the normal derivatives $(\partial u / \partial n)_{+}-(\partial u / \partial n)_{-}+h u_{+}=f$ are given on cracks. The solution is looked for in the form of the sum of a modified single-layer potential and a double-layer potential. The solution of the corresponding integral equation is constructed.

Copyright (c) 2006 Hindawi Publishing Corporation. All rights reserved.

\section{Introduction}

Krutitskii studied in [6] the boundary value problem for the Helmholtz equation outside several cuts in the plane. Two boundary conditions were given on the cuts. One of them specified the jump of the unknown function. Another one of the type of the Robin condition contained the jump of the normal derivative of an unknown function and the one-side limit of this function on the cuts. He looked for a solution of the problem in the form of the sum of a single-layer potential and an angular potential. He reduced the problem to the solution of a uniquely solvable integral equation. So, he proved the unique solvability of the problem.

This paper studies the boundary value problem for the Laplace equation in a bounded planar domain with several cuts inside. The cuts in [6] are smooth open arcs. The cracks in this paper are arbitrary closed subsets of such sets. The Robin condition is given on the boundary of the domain. The same conditions as that in [6] are on the cuts. The case when the jumps of the solution and its normal derivatives are given on cracks is included. So, this problem is a generalization of the problem studied in $[4,5]$. I looked for a solution in a similar form like in [6] but instead of a single-layer potential, I used a modified single-layer potential. I reduced the problem to the solution of an integral equation and constructed the solution of this equation. So, I constructed a solution of the problem which is a new result even if there are no cuts inside the domain. 


\section{Formulation of the problem}

Let $M \subset \mathbb{R}^{m}$. We denote by $C^{0}(M)$ the set of all continuous functions on $M$. If $k$ is a positive integer, denote by $C^{k}(M)$ the set of all functions $f$ such that there is continuous $D^{\alpha} f$ on $M$ for each multi-index $\alpha$ with the length at most $k$. If $0<\beta<1$, denote by $C^{\beta}(M)$ the set of $\beta$-Hölder functions on $M$, that is, the set of continuous functions $f$ on $M$ such that

$$
\sup _{x \in M}|f(x)|+\sup _{x, y \in M ; x \neq y} \frac{|f(x)-f(y)|}{|x-y|^{\beta}}<\infty .
$$

If $k$ is a positive integer and $0<\beta<1$, denote by $C^{k+\beta}(M)$ the set of all functions $f \in$ $C^{k}(M)$ such that $D^{\alpha} f \in C^{\beta}(M)$ for each multi-index $\alpha$ with the length $k$.

We say that a bounded open set $H \subset \mathbb{R}^{2}$ has $C^{\alpha}$ boundary $\partial H$ if there exist a finite number of "local" coordinate systems $\left(x_{k}, y_{k}\right)(k=1, \ldots, m)$ and a finite number of functions $\varphi_{k}$ of class $C^{\alpha}, k=1,2, \ldots, m$, defined on $(-\delta, \delta)$ (where $\left.\delta>0\right)$ such that

(1) $\left(x_{k}, y_{k}\right) \in H$ for $\left|x_{k}\right|<\delta, \varphi_{k}\left(x_{k}\right)-\delta<y_{k}<\varphi_{k}\left(x_{k}\right)$,

(2) $\left(x_{k}, y_{k}\right) \notin \mathrm{cl} H$, the closure of $H$, for $\left|x_{k}\right|<\delta, \varphi_{k}\left(x_{k}\right)<y_{k}<\varphi_{k}\left(x_{k}\right)+\delta$,

(3) for every $z \in \partial H$, there exist $k(k=1, \ldots, m)$ and $x_{k} \in(-\delta, \delta)$ such that $z=\left(x_{k}\right.$, $\left.\varphi_{k}\left(x_{k}\right)\right)$ in the corresponding coordinate system.

Let $H, H_{+} \subset \mathbb{R}^{2}$ be bounded open sets with $C^{1}$ boundaries and let $\mathrm{cl} H_{+} \subset H, H$ be connected. Put $H_{-}=H \backslash \mathrm{cl} H_{+}$. Denote by $n(x)\left(n^{+}(x), n^{-}(x)\right)$ the unit exterior normal of $H\left(H_{+}, H_{-}\right)$at $x$, respectively. For $\Gamma$, the closed subset of $\partial H_{+}$, put $G=H \backslash \Gamma$. If $u$ is a function in $G, x \in \partial G\left(x \in \partial H_{+}, x \in \partial H_{-}\right)$, denote by $u(x)\left(u_{+}(x), u_{-}(x)\right)$ the limit of $u$ at $x$ with respect to $G\left(H_{+}, H_{-}\right)$, respectively. We will study the Robin problem for the Laplace equation on the cracked open set $G$.

Let $h \in C^{0}(\partial G), h \geq 0, f \in C^{0}(\partial G), g \in C^{0}(\Gamma)$. We say that a function $u$ in $G$ is a solution of the problem

$$
\begin{gathered}
\Delta u=0 \quad \text { in } G, \\
\left(\frac{\partial u}{\partial n}\right)+h u=f \quad \text { on } \partial G \backslash \Gamma, \\
u_{+}-u_{-}=g \quad \text { on } \Gamma, \\
{\left[\frac{\partial u}{\partial n^{+}}\right]_{+}-\left[\frac{\partial u}{\partial n^{+}}\right]_{-}+h u_{+}=f \quad \text { on } \Gamma,}
\end{gathered}
$$

if

(1) $u$ is harmonic in $G$;

(2) there is an extension of $u$ onto the function from $\mathrm{C}^{1}\left(\mathrm{cl}_{+}\right)$;

(3) there is an extension of $u$ onto the function from $C^{1}\left(\mathrm{cl}_{-}\right)$;

(4) $n(x) \cdot \nabla u(x)+h(x) u(x)=f(x)$ in $\partial G \backslash \Gamma$;

(5) $u_{+}(x)-u_{-}(x)=g(x)$ in $\Gamma$;

(6) $n^{+}(x) \cdot[\nabla u]_{+}(x)-n^{+}(x) \cdot[\nabla u]_{-}(x)+h(x) u_{+}(x)=f(x)$ in $\Gamma$.

If $h=0$, we will talk about the Neumann problem. In the opposite case, we will talk about the Robin problem. 


\section{Uniqueness}

Denote by $\mathscr{H}_{k}$ the $k$-dimensional Hausdorff measure normalized so that $\mathscr{H}_{k}$ is the Lebesgue measure in $\mathbb{R}^{k}$.

Theorem 3.1. Let $h \in C^{0}(\partial G), h \geq 0, f \equiv 0, g \equiv 0$, and let $u$ be a solution of the problem (2.2). Then $u$ is constant in $G$. If there is $x \in \partial G$ so that $h(x)>0$, then $u=0$ in $G$.

Proof. $u \in C^{0}(\mathrm{cl} G)$ because $g \equiv 0$. Since $n^{-}=-n^{+}$on $\partial H_{+}$, we get, using Green's formula for $H_{+}$and $H_{-}$,

$$
\begin{aligned}
0 & =\int_{\partial H}\left[u(n \cdot \nabla u)+h u^{2}\right] d \mathscr{H}_{1}+\int_{\Gamma} u\left\{n^{+} \cdot[\nabla u]_{+}-n^{+} \cdot[\nabla u]_{-}+h u_{+}\right\} d \mathscr{H}_{1} \\
& =\int_{\partial G} h u^{2} d \mathcal{H}_{1}+\int_{\partial H_{+}} u\left(n^{+} \cdot[\nabla u]_{+}\right) d \mathcal{H}_{1}+\int_{\partial H_{-}} u\left(n^{-} \cdot[\nabla u]_{-}\right) d \mathcal{H}_{1} \\
& =\int_{\partial G} h u^{2} d \mathcal{H}_{1}+\int_{G}|\nabla u|^{2} d \mathcal{H}_{2} .
\end{aligned}
$$

Since $h \geq 0$, we have

$$
\int_{\partial G} h u^{2} d \mathscr{H}_{1}=0, \quad \int_{G}|\nabla u|^{2} d \mathscr{H}_{2}=0
$$

Since $\nabla u=0$ in $G, u$ is constant in each component of $G$. Since $u \in C^{0}(H)$ and $H$ is connected, there is a constant $c$ such that $u=c$ on $H$. If there is $x \in \partial G$ so that $h(x)>$ 0 , then $0=n^{+}(x) \cdot[\nabla u]_{+}(x)-n^{+}(x) \cdot[\nabla u]_{-}(x)+h(x) u_{+}(x)=h(x) c$. Therefore, $c=0$.

\section{Necessary conditions for the solvability}

Let $K$ be a closed subset of $\partial H_{-}$. For a function $f$ defined on $K$, denote $f_{K}=f$ on $K$, $f_{K}=0$ on $\partial H_{-} \backslash K$. We say that $f \in C_{0}^{\alpha}(K)$ if $f_{K} \in C^{\alpha}\left(\partial H_{-}\right)$. If $0<\alpha<1$, denote

$$
\begin{gathered}
\|f\|_{C_{0}^{\alpha}(K)}=\sup _{x \in K}|f(x)|+\sup _{x, y \in K ; x \neq y} \frac{|f(x)-f(y)|}{|x-y|^{\alpha}}, \\
\|f\|_{C_{0}^{1+\alpha}(K)}=\sup _{x \in K}|f(x)|+\left\|\frac{\partial f_{K}}{\partial \tau}\right\|_{C_{0}^{\alpha}(K)},
\end{gathered}
$$

where $\tau(x)=\left(-n_{2}(x), n_{1}(x)\right)$ is the unit tangential vector of $H_{-}$at $x$.

Proposition 4.1. Let $h \in C^{0}(\partial G), h \geq 0, f \in C^{0}(\partial G), g \in C^{0}(\Gamma)$, and let $u$ be a solution of the problem (2.2). Then $g \in C_{0}^{1}(\Gamma)$. If $h \in C_{0}^{0}(\partial G)$, then $f \in C_{0}^{0}(\partial G)$. If $h \equiv 0$, then

$$
\int_{\partial G} f d \mathscr{H}_{1}=0 .
$$

Proof. Since $g_{\Gamma}=u_{+}-u_{-}$in $\partial H_{+}$and $u_{+}, u_{-} \in C^{1}\left(\partial H_{+}\right)$, we deduce that $g_{\Gamma} \in C^{1}\left(\partial H_{+}\right)$. If $h \equiv 0$, we get, using Green's theorem and the fact that $n^{-}=-n^{+}$on $\partial H_{+}$,

$$
\int_{\partial G} f d \mathscr{H}_{1}=\int_{\partial H_{+}} n^{+} \cdot[\nabla u]_{+} d \mathscr{H}_{1}+\int_{\partial H_{-}} n^{-} \cdot[\nabla u]_{-} d \mathscr{H}_{1}=0 .
$$


Suppose now that $h \in C_{0}^{0}\left(\partial H_{+}\right)$. Since $f_{\partial G}=n^{+} \cdot[\nabla u]_{+}-n^{+} \cdot[\nabla u]_{-}+h u_{+}$in $\partial H_{+}$and $u_{+}, n^{+},[\nabla u]_{+},[\nabla u]_{-} \in C^{0}\left(\partial H_{+}\right)$, we get $f_{\partial G} \in C^{0}\left(\partial H_{+}\right)$.

\section{Single-layer potentials}

Fix $R>0$. For $f \in C^{0}(\partial G)$, define

$$
\mathscr{S}_{R} f(x)=(2 \pi)^{-1} \int_{\partial G} f(y) \log \left(\frac{R}{|x-y|}\right) d \mathscr{H}_{1}(y)
$$

as the modified single-layer potential with density $f$. In the case of several sets, we will write $\mathscr{Y}_{R}^{G} f$. (For $R=1$, we get the usual single-layer potential.) Note that $\mathscr{Y}_{R} f$ differs by constants. The function $\mathscr{Y}_{R} f$ is harmonic in $\mathbb{R}^{2} \backslash \partial G$.

Lemma 5.1. Let $H, H_{+}$have boundary of class $C^{1+\gamma}$, where $0<\gamma<1$. Let $0<\beta<\gamma, R>0$. If $f \in C_{0}^{\beta}(\partial G)$, then $\mathscr{S}_{R} f \in C^{0}\left(\mathbb{R}^{2}\right), \mathscr{Y}_{R} f \in C^{1}\left(\mathrm{cl} H_{+}\right), \mathscr{Y}_{R} f \in C^{1}\left(\mathrm{cl} H_{-}\right)$, and

$$
\sup _{x \in G}\left|\mathscr{I}_{R} f(x)\right|+\sup _{x \in G}\left|\nabla \mathscr{Y}_{R} f(x)\right| \leq M\|f\|_{C_{0}^{\beta}(\partial G)}
$$

where $M$ depends on $G, R$, and $\beta$.

Proof. According to [3, Lemma 2.18], the function $\mathscr{S}_{R} f$ is continuous in $\mathbb{R}^{2}$. According to [10, page 227], we have $\mathscr{S}_{R} f \in C^{1}\left(\mathrm{cl} H_{+}\right), \mathscr{S}_{R} f \in C^{1}\left(\mathrm{cl} H_{-}\right)$. Thus $\mathscr{S}_{R}: f \mapsto \mathscr{S}_{R} f$ is a linear operator from $C_{0}^{\beta}(\partial G)$ to $C^{1}\left(\mathrm{cl}_{-}\right)$. Easy calculation yields that this operator is closed. According to the closed graph theorem (see [13, Chapter II, Section 6, Theorem $1]$ ), it is bounded, similarly for $H_{+}$.

Lemma 5.2. Let $H, H_{+}$have boundary of class $C^{1+\gamma}$, where $0<\gamma<1$. Let $0<\beta<\gamma, R>0$, $h \in C_{0}^{\beta}(\partial G)$. If $V_{h} f=h S_{R} f$ for $f \in C_{0}^{\beta}(\partial G)$ is denoted, then $V_{h}$ is a compact linear operator on $C_{0}^{\beta}(\partial G)$.

Proof. Lemma 5.1 yields that $\mathscr{S}_{R}$ represents a bounded linear operator from $C_{0}^{\beta}(\partial G)$ to $C^{1}\left(\mathrm{cl}_{-}\right)$. Since the identity operator is a compact operator from $C^{1}\left(\mathrm{cl}_{-}\right)$to $C^{\beta}\left(\partial H_{-}\right)$, the operator $\mathscr{S}_{R}$ is a compact linear operator from $C_{0}^{\beta}(\partial G)$ to $C^{\beta}\left(\partial H_{-}\right)$by [13, Chapter $\mathrm{X}$, Section 2]. Since $h_{\partial G} \in C^{\beta}\left(\partial H_{-}\right)$, the operator $H: g \mapsto h g$ is a bounded linear operator in $C^{\beta}\left(\partial H_{-}\right)$. Since $H \mathscr{S}_{R}$ is a compact linear operator from $C_{0}^{\beta}(\partial G)$ to $C^{\beta}\left(\partial H_{-}\right)$by [13, Chapter X, Section 2], the operator $V_{h}$ is a compact linear operator on $C_{0}^{\beta}(\partial G)$.

Lemma 5.3. Let $\varphi \in C_{0}^{0}(\partial G), R>\operatorname{diam} G$, the diameter of $G$. If there is $y \in \partial G$ such that $\varphi(y) \neq 0$, then

$$
0<\int_{\partial G} \varphi(x) S_{R} \varphi(x) d \mathscr{H}_{1}(x)<\infty
$$

Proof. Fix $x_{0}$ in $\partial G$. Put $P(x)=\left(x-x_{0}\right) / R, P_{-1}(x)=R x+x_{0}$ for $x \in \mathbb{R}^{2}, \widetilde{G}=P(G)$. For $x \in \partial \tilde{G}$, put $\tilde{\varphi}(x)=\varphi\left(P_{-1}(x)\right)$. Then $\tilde{\varphi} \in C^{0}(\partial \widetilde{G})$ and $\mathscr{S}_{R} \varphi(x)=R \mathscr{S}_{1}^{\tilde{G}} \tilde{\varphi}(P(x))$ for $x \in$ $G$. Denote by $\mathscr{H}$ the restriction of $\mathscr{H}_{1}$ onto $\partial \widetilde{G}$. Since $\mathscr{Y}_{1}^{\widetilde{G}}|\tilde{\varphi}|$ is continuous in $\mathbb{R}^{2}$ by 
[10, Lemma 4], we conclude that

$$
\int|\widetilde{\varphi}| \mathscr{Y}_{1}^{\widetilde{G}}|\tilde{\varphi}| d \mathscr{H}<\infty
$$

and thus the real measure $\tilde{\varphi} \mathscr{H}$ has finite energy (see [7, Chapter 1, Section 4]). Since $\mathscr{H}_{1}(\{x \in \partial \tilde{G} ;|\tilde{\varphi}(x)|>0\})>0,[7$, Theorem 1.16] yields

$$
\int \tilde{\varphi} \mathscr{S}_{1}^{\tilde{G}} \tilde{\varphi} d \mathscr{H}>0
$$

Now we use the fact that

$$
\int_{\partial G} \varphi(x) S_{R} \varphi(x) d \mathscr{H}_{1}(x)=R^{2} \int_{\partial \widetilde{G}} \tilde{\varphi}(x) S_{1}^{\widetilde{G}} \tilde{\varphi}(x) d \mathscr{H}_{1}(x) .
$$

\section{Double-layer potentials}

If $g \in C_{0}^{0}(\Gamma)$, denote, for $x \in \mathbb{R}^{2} \backslash \Gamma$,

$$
\mathscr{D} g(x)=(2 \pi)^{-1} \int_{\Gamma} g(y)|x-y|^{-2} n^{+}(y) \cdot(y-x) d \mathscr{H}_{1}(y)
$$

as the double-layer potential with density $g$.

If $V$ is a bounded open set with $C^{1}$ boundary, $g \in C(\partial V)$, and $n^{V}(y)$ denotes the exterior unit normal of $V$ at $y$, define on $\mathbb{R}^{2} \backslash \partial V$

$$
\mathscr{D}_{V} g(x)=(2 \pi)^{-1} \int_{\partial V} g(y)|x-y|^{-2} n^{V}(y) \cdot(y-x) d \mathscr{H}_{1}(y)
$$

as the double-layer potential corresponding to $V$ with density $g$.

Lemma 6.1. Let $H, H_{+}$have boundary of class $C^{1+\gamma}$, where $0<\gamma<1$. Let $0<\beta<\gamma, g \in$ $C_{0}^{1+\beta}(\Gamma)$. Then $\mathscr{D g}$ is a harmonic function in $\mathbb{R}^{2} \backslash \Gamma$, $\mathscr{D} g \in C^{1}\left(\mathrm{cl} H_{+}\right), \mathscr{D g} \in C^{1}\left(\mathrm{cl} H_{-}\right)$, $[\mathscr{D} g]_{+}(x)-[\mathscr{D} g]_{-}(x)=g(x)$ on $\Gamma, n^{+}(x) \cdot[\nabla \mathscr{D} g]_{+}(x)-n^{+}(x) \cdot[\nabla \mathscr{D} g]_{-}(x)=0$ on $\Gamma$, and

$$
\sup _{x \in G}|\mathscr{D}(x)|+\sup _{x \in G}|\nabla \mathscr{D} g(x)| \leq M\|g\|_{C_{0}^{1+\beta}(\partial G)},
$$

where $M$ depends on $G, R$, and $\beta$.

Proof. Easy calculations yield that $\mathscr{D} g$ is a harmonic function in $\mathbb{R}^{2} \backslash \Gamma$. Since $\mathscr{D} g=$ $\mathscr{D}_{H_{+}} g=\mathscr{D}_{H_{-}} g$, [9, Theorem 1] yields that $\mathscr{D} g \in C^{0}\left(\mathrm{cl} H_{+}\right), \mathscr{D} g \in C^{0}\left(\mathrm{cl} H_{-}\right)$, and $[\mathscr{D}]_{+}(x)$ $-[\mathscr{D} g]_{-}(x)=g(x)$ in $\Gamma$.

The boundary of $H_{+}$is formed by finitely many Jordan curves. Fix one of these curves $T$. Denote $g_{T}=g$ on $T, g_{T}=0$ elsewhere. Let $T$ be parametrized by the arc length $s: T=\{\varphi(s) ; s \in[a, b]\}$, and $H_{+}$is to the right when the parameter $s$ increases on $T$. Put $f(\varphi(s))=-[g(\varphi)]^{\prime}(s)$. Then $f \in C^{\beta}(T)$ because $g \in C^{1+\beta}\left(\partial H_{+}\right)$. For $x \in \mathbb{R}^{2} \backslash T$ and $s \in[a, b]$, denote by $v(x, \varphi(s))$ the increment of the argument of $y-x$ along the curve 
$\{y=\varphi(t) ; t \in[a, s]\}$, and

$$
V f(x)=(2 \pi)^{-1} \int_{T} v(x, y) f(y) d \mathscr{H}_{1}(y)
$$

is the angular potential corresponding to $f$. Define $f=0$ on $\mathbb{R}^{2} \backslash T$. Since $V f$ is a conjugate function to $-\mathscr{S}_{1}^{H_{+}} f$ (see [10, pages 226-227]), we have $\partial V f / \partial x_{1}=-\partial \varphi_{1}^{H_{+}} f / \partial x_{2}$, $\partial V f / \partial x_{2}=\partial \varphi_{1}^{H_{+}} f / \partial x_{1}$. Lemma 5.1 gives $\nabla V f \in C^{0}\left(\mathrm{cl} H_{+}\right), \nabla V f \in C^{0}\left(\mathrm{cl} H_{-}\right)$. Using boundary properties of single-layer potentials (see [2, Theorem 2.2.13]), we can deduce that $n^{+}(x) \cdot[\nabla V f]_{+}(x)-n^{+}(x) \cdot[\nabla V f]_{-}(x)=0$ in T. Put $\tilde{g}=g-g(\varphi(a)), \hat{g}=g(\varphi(a))$ on $T, \tilde{g}, \hat{g}=0$ elsewhere. Since

$$
g_{T}(\varphi(s))-g_{T}(\varphi(a))=\int_{a}^{s}(-f(t)) d t=\int_{s}^{b} f(t) d t,
$$

we have $\mathscr{D}_{H_{+}} \tilde{g}=V f$ by [10, page 226]. Therefore $\mathscr{D}_{H_{+}} \tilde{g} \in C^{1}\left(\mathrm{cl} H_{+}\right), \mathscr{D}_{H_{+}} \tilde{g} \in C^{1}\left(\mathrm{cl}_{-}\right)$, and $n^{+}(x) \cdot\left[\nabla \mathscr{D}_{H_{+}} \tilde{g}\right]_{+}(x)-n^{+}(x) \cdot\left[\nabla \mathscr{D}_{H_{+}} \tilde{g}\right]_{-}(x)=0$ in T. According to [9, page 137], the function $\mathscr{D} \hat{g}$ is constant in the interior of $T$ and in the exterior of $T$ as well. Thus $\nabla \mathscr{D}_{H_{+}} \hat{g}=0$ in $\mathbb{R}^{2} \backslash T$. So, $\mathscr{D} g_{T}=\mathscr{D}_{H_{+}} \tilde{g}+\mathscr{D}_{H_{+}} \hat{g} \in C^{1}\left(\operatorname{cl} H_{+}\right) \cap C^{1}\left(\mathrm{cl}_{-}\right)$and $n^{+}(x)$. ${ }_{+}(x)-n^{+}(x) \cdot\left[\nabla \mathscr{D} g_{T}\right]_{-}(x)=0$ on $\partial H_{+}$. Summing over all $T$, we get $\mathscr{D} g \in C^{1}\left(\mathrm{cl} H_{+}\right)$, $\mathscr{D} g \in C^{1}\left(\mathrm{cl} H_{-}\right)$, and $n^{+}(x) \cdot[\nabla \mathscr{D} g]_{+}(x)-n^{+}(x) \cdot[\nabla \mathscr{D} g]_{-}(x)=0$ on $\Gamma$.

Since $\mathscr{D}: g \mapsto \mathscr{D} g$ is a closed linear operator from $C_{0}^{1+\beta}(\Gamma)$ to $C^{1}\left(\mathrm{cl} H_{+}\right)$, it is bounded by the closed graph theorem (see [13, Chapter II, Section 6, Theorem 1]), similarly for $H_{-}$.

\section{Reduction of the problem}

Let $H, H_{+}$have boundary of class $C^{1+\gamma}$, where $0<\gamma<1$. Let $0<\beta<\gamma, f \in C_{0}^{\beta}(\partial G), h \in$ $C_{0}^{\beta}(\partial G), h \geq 0, g \in C_{0}^{1+\beta}(\Gamma)$. We will look for a solution $u$ of the problem (2.2) in the form

$$
u=\mathscr{D} g+v
$$

According to Lemma 6.1 and [9, Theorem 1], the function $u$ is a solution of the problem (2.2) if and only if the function $v$ is a solution of the problem

$$
\begin{gathered}
\Delta v=0 \quad \text { in } G \\
\frac{\partial v}{\partial n}+h v=F \quad \text { on } \partial G \backslash \Gamma, \\
v_{+}-v_{-}=0 \quad \text { on } \Gamma, \\
{\left[\frac{\partial v}{\partial n^{+}}\right]_{+}-\left[\frac{\partial v}{\partial n^{+}}\right]_{-}+h v_{+}=F \quad \text { on } \Gamma}
\end{gathered}
$$

where

$$
\begin{gathered}
F=f-\frac{\partial \mathscr{D} g}{\partial n}-h \mathscr{g} \quad \text { on } \partial G \backslash \Gamma, \\
F(x)=f(x)-h(x)\left(\frac{1}{2} g(x)+\int_{\Gamma} \frac{n^{+}(y) \cdot(y-x)}{2 \pi|x-y|^{2}} g(y) d \mathscr{H}_{1}(y)\right), \quad x \in \Gamma .
\end{gathered}
$$


$F \in C_{0}^{\beta}(\partial G)$ because $f \in C_{0}^{\beta}(\partial G), h \in C_{0}^{\beta}(\partial G), \mathscr{D} g \in C^{\infty}\left(\mathbb{R}^{2} \backslash \Gamma\right), \mathscr{D} g \in C^{1}\left(\mathrm{cl} H_{+}\right)$by Lemma 6.1, and

$$
[\mathscr{D} g]_{+}(x)=\frac{1}{2} g(x)+\frac{1}{2 \pi} \int_{\Gamma} \frac{n^{+}(y) \cdot(y-x)}{|x-y|^{2}} g(y) d \mathscr{H}_{1}(y)
$$

for $x \in \Gamma$ by [1, Theorem].

We will look for a solution of the problem (7.2) in the form of a modified single-layer potential $\mathscr{I}_{R} w$, where $w \in C_{0}^{\beta}(\partial G)$ and $R>\operatorname{diam} G$.

Define for $w \in C_{0}^{\beta}(\partial G), x \in \partial H$,

$$
K_{G}^{*} w(x)=(2 \pi)^{-1} \int_{\partial G} w(y)|x-y|^{-2} n(x) \cdot(y-x) d \mathscr{H}_{1}(y) .
$$

According to [9, Theorem 2],

$$
\begin{gathered}
n(x) \cdot \nabla \mathscr{Y}_{R} w(x)=\frac{w(x)}{2}+K_{G}^{*} w(x) \quad \text { for } x \in \partial G \backslash \Gamma, \\
n^{+}(x) \cdot\left[\nabla \mathscr{S}_{R} w\right]_{+}(x)-n^{+}(x) \cdot\left[\nabla \mathscr{Y}_{R} w\right]_{-}(x)=w(x) \quad \text { in } \Gamma .
\end{gathered}
$$

Since the modified single layer potential $\mathscr{S}_{R} w$ is a harmonic function in $G$, we get using Lemma 5.1 that $\mathscr{Y}_{R} w$ is a solution of the problem (7.2) if and only if

$$
T_{h, R} w=F,
$$

where

$$
\begin{gathered}
T_{h, R} w=\frac{w}{2}+K_{G}^{*} w+h \mathscr{S}_{R} w \quad \text { on } \partial G \backslash \Gamma, \\
T_{h, R} w=w+h \mathscr{Y}_{R} w \quad \text { on } \Gamma .
\end{gathered}
$$

\section{Solvability of the problem}

Lemma 8.1. Let $H, H_{+}$have boundary of class $C^{1+\gamma}$, where $0<\gamma<1$. Let $0<\beta<\gamma, h \in$ $C_{0}^{\beta}(\partial G), h \geq 0, R>\operatorname{diam} G$. Then $K_{G}^{*}$ is a compact linear operator from $C_{0}^{\beta}(\partial G)$ to $C^{\beta}(\partial H)$ and $T_{h, R}$ is a bounded linear operator in $C_{0}^{\beta}(\partial G)$.

Proof. Fix $\alpha \in(\beta, \gamma)$. Then $K_{H}^{*}$ is a linear operator from $C^{\beta}(\partial H)$ to $C^{\alpha}(\partial H)$ by [11, Theorem 14.IV]. Since $K_{H}^{*}$ is a bounded linear operator from $C^{\beta}(\partial H)$ to $C^{0}(\partial H)$ by $(7.7)$ and Lemma 5.1, the operator $K_{H}^{*}$ is a closed operator from $C^{\beta}(\partial H)$ to $C^{\alpha}(\partial H)$. This gives that the operator $K_{H}^{*}$ is a closed linear operator from $C_{0}^{\beta}(\partial G)$ to $C^{\alpha}(\partial H)$. The closed graph theorem (see [13, Chapter II, Section 6, Theorem 1]) shows that $K_{H}^{*}$ is a bounded linear operator from $C_{0}^{\beta}(\partial G)$ to $C^{\alpha}(\partial H)$. Since the identity operator is a compact operator from $C^{\alpha}(\partial H)$ to $C^{\beta}(\partial H)$, the operator $K_{G}^{*}$ is a compact linear operator from $C_{0}^{\beta}(\partial G)$ to $C^{\beta}(\partial H)$ by [13, Chapter X, Section 2]. Since $K_{G}^{*}$ is a bounded linear operator from $C_{0}^{\beta}(\partial G)$ to $C^{\beta}(\partial H)$, the operator $T_{0, R}$ is a bounded linear operator in $C_{0}^{\beta}(\partial G)$. Since $T_{h, R}-T_{0, R}$ is a bounded linear operator in $C_{0}^{\beta}(\partial G)$ by Lemma 5.2, the operator $T_{h, R}$ is a bounded linear operator in $C_{0}^{\beta}(\partial G)$. 
Notation 8.2. Let $X$ be a real Banach space. Denote by compl $X=\{x+i y ; x, y \in X\}$ the complexification of $X$ with the norm $\|x+i y\|=\|x\|+\|y\|$. If $T$ is a bounded linear operator on $X$, we define $T(x+i y)=T x+i T y$ as the bounded linear extension of $T$ onto complX.

Definition 8.3. The bounded linear operator $T$ on the Banach space $X$ is called Fredholm if $\alpha(T)$, the dimension of the kernel of $T$, is finite, the range $T(X)$ of $T$ is a closed subspace of $X$, and $\beta(T)$, the codimension of $T(X)$, is finite. The number $i(T)=\alpha(T)-\beta(T)$ is the index of $T$.

Lemma 8.4. Let $H, H_{+}$have boundary of class $C^{1+\gamma}$, where $0<\gamma<1$. Let $0<\beta<\gamma, h \in$ $C_{0}^{\beta}(\partial G), h \geq 0, R>\operatorname{diam} G$. If $\lambda \in C, \lambda \neq 1 / 2, \lambda \neq 1$, then $T_{h, R}-\lambda I$ is a Fredholm operator with index 0 in $\operatorname{compl} C_{0}^{\beta}(\partial G)$. (Here I denotes the identity operator.)

Proof. Denote $L f(x)=f(x) / 2$ for $x \in \partial H, L f(x)=f(x)$ for $x \in \Gamma$. Then $L-\lambda I$ is a Fredholm operator in $\operatorname{compl} C_{0}^{\beta}(\partial G)$. Since $T_{0, R}-L$ is a compact operator in $C_{0}^{\beta}(\partial G)$ by Lemma 8.1, the operator $T_{0, R}-\lambda I$ is a Fredholm operator with index 0 in $C_{0}^{\beta}(\partial G)$ by [12, Theorem 5.10]. Since $T_{h, R}-T_{0, R}$ is a compact linear operator in $C_{0}^{\beta}(\partial G)$ by Lemma 5.2, the operator $T_{h, R}-\lambda I$ is a Fredholm operator with index 0 in $C_{0}^{\beta}(\partial G)$ by $[12$, Theorem 5.10].

Lemma 8.5. Let $H, H_{+}$have boundary of class $C^{1+\gamma}$, where $0<\gamma<1$. Let $0<\beta<\gamma, R>$ $\operatorname{diam} G, \varphi \in C_{0}^{\beta}(\partial G)$. If $T_{0, R}^{2} \varphi=0$, then $T_{0, R} \varphi=0$.

Proof. According to Section 7, the modified single-layer potential $\mathscr{S}_{R} T_{0, R} \varphi$ is a solution of the problem (2.2) with $h \equiv 0, g \equiv 0$, and $f=T_{0, R}^{2} \varphi=0$. According to Theorem 3.1, there is a constant $c$ such that $\mathscr{Y}_{R} T_{0, R} \varphi=c$ on $G$.

According to Section 7, the modified single-layer potential $\mathscr{S}_{R} \varphi$ is a solution of the problem (2.2) with $h \equiv 0, g \equiv 0$, and $f=T_{0, R} \varphi$. Thus

$$
\int_{\partial G} T_{0, R} \varphi=0
$$

by proposition 4.1. Since $\mathscr{Y}_{R} T_{0, R} \varphi=c$ in $G$ and $\mathscr{Y}_{R} T_{0, R} \varphi$ is continuous in $\mathbb{R}^{2}$ by Lemma 5.1 , we obtain

$$
\int_{\partial G}\left(T_{0, R} \varphi\right) \mathscr{T}_{R} T_{h, R} \varphi d \mathscr{H}_{1}=c \int_{\partial G} T_{0, R} \varphi=0 .
$$

According to Lemma 5.3, we have $T_{0, R} \varphi=0$ a.e. in $\partial G$.

Proposition 8.6. Let $H, H_{+}$have boundary of class $C^{1+\gamma}$, where $0<\gamma<1$. Let $0<\beta<\gamma$, $h \in C_{0}^{\beta}(\partial G), h \geq 0, h(x)>0$ for some $x \in \partial G, R>\operatorname{diam} G$. Then $T_{h, R}$ is continuosly invertible in $C_{0}^{\beta}(\partial G)$. Denote by $C_{0,0}^{\beta}(\partial G)$ the space of all $f \in C_{0}^{\beta}(\partial G)$ for which (4.2) holds. Then $T_{0, R}$ is continuously invertible in $C_{0,0}^{\beta}(\partial G)$.

Proof. Let $\varphi \in C_{0}^{\beta}(\partial G)$ be such that $\mathscr{I}_{R} \varphi=0$ in $G$. Then $\mathscr{S}_{R} \varphi=0$ in $\partial G$ by Lemma 5.1. According to Lemma 5.3, we have $\varphi \equiv 0$. 
If $\varphi \in C_{0}^{\beta}(\partial G)$ and $T_{h, R} \varphi=0$, then $\mathscr{T}_{R} \varphi$ is a solution of the problem (2.2) with $f \equiv 0$, $g \equiv 0$ by Section 7. Since $\mathscr{S}_{R} \varphi=0$ in $G$ by Theorem 3.1, we have $\varphi \equiv 0$. Since $T_{h, R}$ is a Fredholm operator in $C_{0}^{\beta}(\partial G)$ with index 0 by Lemma 8.4, we obtain $T_{h, R}\left(C_{0}^{\beta}(\partial G)\right)=$ $C_{0}^{\beta}(\partial G)$. Thus, $T_{h, R}$ is continuously invertible in $C_{0}^{\beta}(\partial G)$ by [13, Chapter II, Section 6 , Proposition 3] and [13, Chapter II, Section 6, Theorem 1].

If $\varphi \in C_{0}^{\beta}(\partial G)$, then $\mathscr{S}_{R} \varphi$ is a solution of the problem (2.2) with $h \equiv 0, g \equiv 0$, and $f=$ $T_{0, R} \varphi$ (see Section 7). Thus $T_{0, R} \varphi \in C_{0,0}^{\beta}(\partial G)$ by Proposition 4.1. Hence, $T_{0, R}\left(C_{0}^{\beta}(\partial G)\right) \subset$ $C_{0,0}^{\beta}(\partial G)$ and $\beta\left(T_{0, R}\right) \geq 1$. Since $T_{0, R}$ is a Fredholm operator in $C_{0}^{\beta}(\partial G)$ with index 0 by Lemma 8.4, there is a nontrivial $\varphi_{1} \in C_{0}^{\beta}(\partial G)$ such that $T_{0, R} \varphi_{1}=0$. According to Section 7 and Theorem 3.1, there is a constant $c_{1}$ such that $\mathscr{S}_{R} \varphi_{1}=c_{1}$ in $G$. Since $c_{1}=0$ yields $\varphi_{1}=0$ we deduce that $c_{1} \neq 0$. If $\varphi \in C_{0}^{\beta}(\partial G), T_{0, R} \varphi=0$, then Section 7 and Theorem 3.1 imply that there is a constant $c$ such that $\mathscr{S}_{R} \varphi=c$ in $G$. Since $\mathscr{S}_{R}\left(\varphi-\left(c / c_{1}\right) \varphi_{1}\right)=0$ in $G$, we obtain that $\varphi-\left(c / c_{1}\right) \varphi_{1} \equiv 0$. This means that $\alpha\left(T_{0, R}\right)=1$. Since $T_{0, R}\left(C_{0}^{\beta}(\partial G)\right) \subset C_{0,0}^{\beta}(\partial G)$, $\beta\left(T_{0, R}\right)=\alpha\left(T_{0, R}\right)=1$ shows that $T_{0, R}\left(C_{0}^{\beta}(\partial G)\right)=C_{0,0}^{\beta}(\partial G)$.

According to Lemma 8.5, the operator $T_{0, R}$ is injective in $T_{0, R}\left(C_{0}^{\beta}(\partial G)\right)=C_{0,0}^{\beta}(\partial G)$. Since $C_{0,0}^{\beta}(\partial G)$ is a $T_{0, R}$-invariant closed linear subspace of finite codimension in $C_{0}^{\beta}(\partial G)$ and $T_{0, R}$ is a Fredholm operator with index 0 , the restriction of $T_{0, R}$ onto $C_{0,0}^{\beta}(\partial G)$ is a Fredholm operator with index 0 by [8, Proposition 3.7.1.]. Since $T_{0, R}$ is injective in $C_{0,0}^{\beta}(\partial G)$, it is surjective in $C_{0,0}^{\beta}(\partial G)$. The closed graph theorem (see [13, Chapter II, Section 6, Theorem 1]) gives that $T_{0, R}$ is continuously invertible in $C_{0,0}^{\beta}(\partial G)$.

Theorem 8.7. Let $H, H_{+}$have boundary of class $C^{1+\gamma}$, where $0<\gamma<1$. Let $0<\beta<\gamma, h \in$ $C_{0}^{\beta}(\partial G), h \geq 0, h(x)>0$ for some $x \in \partial G, f \in C_{0}^{\beta}(\partial G), g \in C_{0}^{1+\beta}(\Gamma)$. Then there is a unique solution of the problem (2.2).

Proof. Fix $R>\operatorname{diam} G$. According to Proposition 8.6, there is $T_{h, R}^{-1}$ in $C_{0}^{\beta}(\partial G)$. Let $F$ be given by (7.3). Then

$$
u=\mathscr{D} g+\mathscr{I}_{R} T_{h, R}^{-1} F
$$

is a solution of the problem (2.2) by Section 7. This solution is unique by Theorem 3.1.

Theorem 8.8. Let $H, H_{+}$have boundary of class $C^{1+\gamma}$, where $0<\gamma<1$. Let $0<\beta<\gamma, h \equiv 0$, $f \in C_{0}^{\beta}(\partial G), g \in C_{0}^{1+\beta}(\Gamma)$. Then there is a solution of the problem (2.2) if and only if (4.2) holds. This solution is unique up to an additive constant.

Proof. If there is a solution of the problem (2.2), the relation (4.2) holds by Proposition 4.1. Suppose now that (4.2) holds. Fix $R>\operatorname{diam} G$. Denote by $T$ the restriction of $T_{0, R}$ onto $C_{0,0}^{\beta}(\partial G)$. Then there is $T^{-1}$ by Proposition 8.6. Let $F$ be given by (7.4). Then $u=$ $\mathscr{D} g+\mathscr{S}_{R} T^{-1} F$ is a solution of the problem (2.2) by Section 7. This solution is unique up to an additive constant by Theorem 3.1. 


\section{Solution of the problem}

Lemma 9.1. Let $H, H_{+}$have boundary of class $C^{1+\gamma}$, where $0<\gamma<1$. Let $0<\beta<\gamma, R>$ $\operatorname{diam} G, h \in C_{0}^{\beta}(\partial G), h \geq 0, f \in \operatorname{compl} C_{0}^{\beta}(\partial G), f(x) \neq 0$ for some $x \in \partial G$. If $\lambda$ is a complex number such that $T_{h, R} f=\lambda f$, then $\lambda \geq 0$.

Proof. Take $f_{1}, f_{2} \in C_{0}^{\beta}(\partial G)$ such that $f=f_{1}+i f_{2}$. Since $\mathscr{S}_{R}\left(f_{1}-i f_{2}\right) \in \operatorname{compl} C^{1}\left(\operatorname{cl} H_{+}\right)$, $\mathscr{Y}_{R}\left(f_{1}-i f_{2}\right) \in \operatorname{compl} C^{1}\left(\mathrm{cl} H_{-}\right), \mathscr{Y}_{R}\left(f_{1}-i f_{2}\right) \in \operatorname{compl} C^{0}\left(\mathbb{R}^{2}\right)$ by Lemma 5.1 , we get using Green's formula that

$$
\begin{aligned}
& \int_{\partial H_{+}}\left[\mathscr{Y}_{R}\left(f_{1}-i f_{2}\right)\right] n^{+} \cdot\left[\nabla \mathscr{Y}_{R}\left(f_{1}+i f_{2}\right)\right]_{+}=\int_{H_{+}}\left[\left|\nabla \mathscr{Y}_{R} f_{1}\right|^{2}+\left|\nabla \mathscr{S}_{R} f_{2}\right|^{2}\right] d \mathscr{H}_{2}, \\
& \int_{\partial H_{-}}\left[\mathscr{Y}_{R}\left(f_{1}-i f_{2}\right)\right] n^{-} \cdot\left[\nabla \mathscr{Y}_{R}\left(f_{1}+i f_{2}\right)\right]_{-}=\int_{H_{-}}\left[\left|\nabla \mathscr{S}_{R} f_{1}\right|^{2}+\left|\nabla \mathscr{Y}_{R} f_{2}\right|^{2}\right] d \mathscr{H}_{2} \text {. }
\end{aligned}
$$

Summing,

$$
\int_{\partial G}\left[\mathscr{Y}_{R}\left(f_{1}-i f_{2}\right)\right] T_{0, R}\left(f_{1}+i f_{2}\right) d \mathscr{H}_{1}=\int_{G}\left[\left|\nabla \mathscr{Y}_{R} f_{1}\right|^{2}+\left|\nabla \mathscr{Y}_{R} f_{2}\right|^{2}\right] d \mathscr{H}_{2} .
$$

Using Fubini's theorem,

$$
\begin{aligned}
\lambda \int_{\partial G}\left(f_{1} \mathscr{Y}_{R} f_{1}+f_{2} \mathscr{Y}_{R} f_{2}\right) d \mathscr{H}_{1}= & \int_{\partial G}\left[\mathscr{Y}_{R}\left(f_{1}-i f_{2}\right)\right] T_{h, R}\left(f_{1}+i f_{2}\right) d \mathscr{H}_{1} \\
= & \int_{G}\left[\left|\nabla \mathscr{Y}_{R} f_{1}\right|^{2}+\left|\nabla \mathscr{S}_{R} f_{2}\right|^{2}\right] d \mathscr{H}_{1} \\
& +\int_{\partial G} h\left[\left(\mathscr{Y}_{R} f_{1}\right)^{2}+\left(\mathscr{Y}_{R} f_{2}\right)^{2}\right] d \mathscr{H}_{1} .
\end{aligned}
$$

Since

$$
0<\int_{\partial G}\left(f_{1} \mathscr{S}_{R} f_{1}+f_{2} \mathscr{S}_{R} f_{2}\right) d \mathscr{H}_{1}<\infty
$$

by Lemma 5.3 and $h \geq 0$, we get

$$
\lambda=\frac{\int_{G}\left[\left|\nabla \mathscr{S}_{R} f_{1}\right|^{2}+\left|\nabla \mathscr{S}_{R} f_{2}\right|^{2}\right] d \mathscr{H}_{1}+\int_{\partial G} h\left[\left(\mathscr{S}_{R} f_{1}\right)^{2}+\left(\mathscr{S}_{R} f_{2}\right)^{2}\right] d \mathscr{H}_{1}}{\int_{\partial G}\left(f_{1} \mathscr{S}_{R} f_{1}+f_{2} \mathscr{S}_{R} f_{2}\right) d \mathscr{H}_{1}} \geq 0 .
$$

Lemma 9.2. Let $H, H_{+}$have boundary of class $C^{1+\gamma}$, where $0<\gamma<1$. Let $0<\beta<\gamma, R>$ $\operatorname{diam} G, f \in \operatorname{compl} C_{0}^{\beta}(\partial G), f(x) \neq 0$ for some $x \in \partial G$. If $\lambda$ is a complex number such that $T_{0, R} f=\lambda f$, then $0 \leq \lambda \leq 1$.

Proof. We can suppose that $\lambda \neq 0$. Lemma 9.1 yields $\lambda>0$ and we thus can suppose that $f \in C_{0}^{\beta}(\partial G)$. Since $\mathscr{I}_{R} \lambda^{-1} f$ is a solution of the problem (2.2) with $h \equiv 0, g \equiv 0$ (see Section 7), Proposition 4.1 gives that $f$ fulfills (4.2). Since $T_{0, R} f=f$ on $\Gamma$, we deduce from $T_{0, R} f=\lambda f$ that $\lambda=1$ or $f=0$ on $\Gamma$. We can restrict ourselves to the case when $f=0$ on $\Gamma$. 
Fix $r>0$ such that $\operatorname{cl} G \subset \Omega_{r}(0) \equiv\left\{y \in \mathbb{R}^{2} ;|x|<r\right\}$ and put $V=\Omega_{r}(0) \backslash \mathrm{cl} G$. Then $\mathscr{S}_{R} f \in C^{1}(\mathrm{cl} V)$ by Lemma 5.1. Using Green's formula,

$$
\begin{aligned}
\int_{V}\left|\nabla \mathscr{Y}_{R} f\right|^{2} d \mathscr{H}_{1} & =\int_{\partial V}\left(\mathscr{Y}_{R} f\right)\left(n \cdot \nabla \mathscr{Y}_{R} f\right) d \mathscr{H}_{1} \\
& =\int_{\partial H}\left(\mathscr{S}_{R} f\right)\left(\frac{1}{2} I-K_{H}^{*}\right) f d \mathscr{H}_{1}+\int_{\partial \Omega_{r}(0)} \frac{\partial \mathscr{Y}_{R} f}{\partial n} \mathscr{Y}_{R} f d \mathscr{H}_{1} \\
& =(1-\lambda) \int_{\partial G} f \mathscr{Y}_{R} f d \mathscr{H}_{1}+\int_{\partial \Omega_{r}(0)} \mathscr{I}_{R} f \frac{\partial \mathscr{S}_{R} f}{\partial n} d \mathscr{H}_{1} .
\end{aligned}
$$

Since (4.2) forces $\mathscr{Y}_{R} f(x)=o(1), \nabla \mathscr{Y}_{R} f(x)=O(1 /|x|)$ as $|x| \rightarrow \infty$, we get for $r \rightarrow \infty$ that

$$
\int_{\mathbb{R}^{2} \backslash \mathrm{cl} G}\left|\nabla \mathscr{S}_{R} f\right|^{2} d \mathscr{H}_{1}=(1-\lambda) \int_{\partial G} f \mathscr{S}_{R} f d \mathscr{H}_{1} .
$$

Using Lemma 5.3, we get

$$
(1-\lambda)=\frac{\int_{\mathbb{R}^{2} \backslash \mathrm{cl} G}\left|\nabla \mathscr{S}_{R} f\right|^{2} d \mathscr{H}_{1}}{\int_{\partial G} f \mathscr{S}_{R} f d \mathscr{H}_{1}} \geq 0 .
$$

So, $\lambda \leq 1$.

Notation 9.3. Let $X$ be a complex Banach space and let $T$ be a bounded linear operator on $X$. Denote by $\sigma(T)$ the spectrum of $T$ and by $r(T)=\sup \{|\lambda| ; \lambda \in \sigma(T)\}$ the spectral radius of $T$.

Lemma 9.4. Let $H, H_{+}$have boundary of class $C^{1+\gamma}$, where $0<\gamma<1$. Let $0<\beta<\gamma, R>$ $\operatorname{diam} G, h \in C_{0}^{\beta}(\partial G), h \geq 0$. Put $V_{h} f=h \mathscr{S}_{R} f$ for $f \in \operatorname{compl} C_{0}^{\beta}(\partial G)$. Then

$$
r\left(V_{h}\right) \leq \sup _{x \in \partial G} S_{R} h(x) .
$$

Proof. Let $\lambda \in \sigma\left(V_{h}\right)$ in $\operatorname{compl} C_{0}^{\beta}(\partial G), \lambda \neq 0$. Since $V_{h}$ is a compact linear operator in $\operatorname{compl} C_{0}^{\beta}(\partial G)$ by Lemma 5.2 , there is $f \in \operatorname{compl} C_{0}^{\beta}(\partial G)$ such that $f(z) \neq 0$ for some $z \in$ $\partial G$ and $V_{h} f=\lambda f$ (see [13, Chapter X, Section 5, Theorem 2]). Using Fubini's theorem,

$$
\begin{aligned}
\int_{\partial G}|\lambda f| d \mathscr{H}_{1} & =\int_{\partial G}\left|h(x) \int_{\partial G}(2 \pi)^{-1} f(y) \log \left(\frac{R}{|x-y|}\right) d \mathscr{H}_{1}(y)\right| d \mathscr{H}_{1}(x) \\
& \leq \iint_{\partial G} h(x)(2 \pi)^{-1}|f(y)| \log \left(\frac{R}{|x-y|}\right) d \mathscr{H}_{1}(x) d \mathscr{H}_{1}(y) \\
& \leq \int_{\partial G}|f| d \mathscr{H}_{1} \sup _{x \in \partial G} \mathscr{Y}_{R} h(x) .
\end{aligned}
$$

Since $f \in \operatorname{compl} C_{0}^{\beta}(\partial G)$ and $f(z) \neq 0$, there is $\delta>0$ such that $|f(x)|>0$ for $x \in \Omega_{\delta}(z) \cap$ $\partial G$ and $\mathcal{H}_{1}\left(\Omega_{\delta}(z) \cap \partial G\right)>0$. Dividing the inequality above by $\int|f|$, we obtain

$$
|\lambda| \leq \sup _{x \in \partial G} S_{R} h(x)
$$


Theorem 9.5. Let $H, H_{+}$have boundary of class $C^{1+\gamma}$, where $0<\gamma<1$. Let $0<\beta<\gamma, h \in$ $C_{0}^{\beta}(\partial G), h \geq 0, f \in C_{0}^{\beta}(\partial G), g \in C_{0}^{1+\beta}(\Gamma)$. If $h \equiv 0$, suppose moreover that (4.2) holds. Put

$$
\alpha_{0} \equiv 1+\sup _{x \in \partial G} \mathscr{I}_{R} h(x)
$$

Fix $\alpha>\alpha_{0} / 2, R>\operatorname{diam} G$. Let $F$ be given by (7.3) and put

$$
\begin{aligned}
\varphi & =\alpha^{-1} \sum_{n=0}^{\infty}\left(I-\alpha^{-1} \widetilde{T}_{h, R}\right)^{n} F, & u=\mathscr{D}+\mathscr{Y}_{R} \varphi, \\
\varphi_{m} & =\alpha^{-1} \sum_{n=0}^{m}\left(I-\alpha^{-1} \widetilde{T}_{h, R}\right)^{n} F, & u_{m}=\mathscr{D} g+\mathscr{Y}_{R} \varphi_{m} .
\end{aligned}
$$

Then $u$ is a solution of the problem (2.2). Moreover, there are constants $q \in(0,1), C \in(1, \infty)$ dependent on $G, h, R$, and $\alpha$ such that

$$
\begin{gathered}
\left\|\alpha^{-1}\left(I-\alpha^{-1} \widetilde{T}_{h, R}\right)^{n} F\right\|_{C_{0}^{\beta}(\partial G)} \leq C q^{n}\left[\|f\|_{C_{0}^{\beta}(\partial G)}+\|g\|_{C_{0}^{1+\beta}(\Gamma)}\right], \\
\sup _{x \in G}|u(x)|+\sup _{x \in G}|\nabla u(x)| \leq C\left[\|f\|_{C_{0}^{\beta}(\partial G)}+\|g\|_{C_{0}^{1+\beta}(\Gamma)}\right], \\
\left.\left\|\varphi-\varphi_{m}\right\|\right|_{C_{0}^{\beta}(\partial G)} \leq C q^{m}\left[\|f\|_{C_{0}^{\beta}(\partial G)}+\|g\|_{C_{0}^{1+\beta}(\Gamma)}\right], \\
\sup _{x \in G}\left|u-u_{m}\right|+\sup _{x \in G}\left|\nabla u(x)-\nabla u_{m}(x)\right| \leq C q^{m}\left[\|f\|_{C_{0}^{\beta}(\partial G)}+\|g\|_{C_{0}^{1+\beta}(\Gamma)}\right] .
\end{gathered}
$$

Proof. If $\lambda \in \sigma\left(T_{0, R}-(1 / 2) I\right), \lambda \neq 0, \lambda \neq 1 / 2$, then $\lambda$ is an eigenvalue of $T_{0, R}-(1 / 2) I$ by Lemma 8.4. Since $|\lambda| \leq 1 / 2$ for each eigenvalue of $T_{0, R}-(1 / 2) I$ by Lemma 9.2, we get $r\left(T_{0, R}-(1 / 2) I\right) \leq 1 / 2$ in compl $C_{0}^{\beta}(\partial G)$. According to Lemma 9.4, [13, Chapter VIII, Section 2], and [13, Satz 45.1 and Lemma 9.4], we have $r\left(T_{h, R}-(1 / 2) I\right) \leq r\left(V_{h}\right)+r\left(T_{0, R}-\right.$ $(1 / 2) I) \leq \alpha_{0}-(1 / 2)$ in $\operatorname{compl} C_{0}^{\beta}(\partial G)$.

If $\lambda \in \sigma\left(T_{h, R}-(1 / 2) I\right), \lambda \neq 0, \lambda \neq 1 / 2$, then $\lambda$ is an eigenvalue of $T_{h, R}$ by Lemma 8.4. Lemma 9.1 forces $\sigma\left(T_{h, R}-(1 / 2) I\right) \subset\left\langle-1 / 2, r\left(T_{h, R}-(1 / 2) I\right)\right\rangle \subset\left\langle-1 / 2, \alpha_{0}-1 / 2\right\rangle$. Using the spectral mapping theorem (see [13, Chapter VIII, Section 7]), we get $\sigma\left(T_{h, R}\right) \subset\left\langle 0, \alpha_{0}\right\rangle$. Put $X=\operatorname{compl} C_{0,0}^{\beta}(\partial G)$ for $h \equiv 0$ and $X=\operatorname{compl} C_{0}^{\beta}(\partial G)$ in the opposite case. Denote by $\tilde{T}_{h, R}$ the restriction of $T_{h, R}$ onto $X$. Let $\lambda \in C \backslash \sigma\left(T_{h, R}\right)$. Since $X$ is a $T_{h, R}$-invariant closed linear subspace of finite codimension in $C_{0}^{\beta}(\partial G)$ (see Section 7 and Proposition 4.1) and $T_{h, R}-\lambda I$ is a Fredholm operator with index 0 , the operator $\widetilde{T}_{h, R}-\lambda I$ is a Fredholm operator with index 0 in $X$ by [8, Proposition 3.7.1.] Since $\widetilde{T}_{h, R}-\lambda I$ is injective, it is surjective and the closed graph theorem (see [13, Chapter II, Section 6, Theorem 1]) gives that $\widetilde{T}_{h, R}-\lambda I$ is continuously invertible. Thus $\sigma\left(\widetilde{T}_{h, R}\right) \subset \sigma\left(T_{h, R}\right) \subset\left\langle 0, \alpha_{0}\right\rangle$. Proposition 8.6 shows that $\sigma\left(\widetilde{T}_{h, R}\right) \subset\left(0, \alpha_{0}\right\rangle$. Using the spectral mapping theorem (see [13, Chapter VIII, Section 7]), we get $\sigma\left(\alpha^{-1} \widetilde{T}_{h, R}-I\right) \subset\{\lambda \in C ;|\lambda|<1\}$. Thus $r\left(\alpha^{-1} \widetilde{T}_{h, R}-I\right)<1$. According to [13, Chapter VIII, Section 2, Theorem 3] and [13, Chapter VIII, Section 2, Theorem 4], 
there are constants $q \in(0,1), M_{1} \in(1, \infty)$ so that

$$
\left\|\left(\alpha^{-1} \widetilde{T}_{h, R}-I\right)^{n}\right\| \leq M_{1} q^{n}
$$

for arbitrary nonnegative integer $n$.

$F \in C_{0}^{\beta}(\partial G)$ by Section 7. Since there is a solution of the problem (2.2) by Theorem 8.7 and Theorem 8.8, there is a solution of the problem (7.2) by Section 7. Thus $F \in X$ by Proposition 4.1. According to (9.15),

$$
\left\|\alpha^{-1}\left(I-\alpha^{-1} \widetilde{T}_{h, R}\right)^{n} F\right\|_{C_{0}^{\beta}(\partial G)} \leq M_{1} q^{n}\|F\|_{C_{0}^{\beta}(\partial G)} .
$$

Hence, $\varphi$ is well defined and

$$
\begin{gathered}
\|\varphi\|_{C_{0}^{\beta}(\partial G)} \leq \sum_{n=0}^{\infty} M_{1} q^{n}\|F\|_{C_{0}^{\beta}(\partial G)} \leq M_{1}(1-q)^{-1}\|F\|_{C_{0}^{\beta}(\partial G)^{\prime}}, \\
\left\|\varphi-\varphi_{m}\right\|_{C_{0}^{\beta}(\partial G)} \leq \sum_{n=m+1}^{\infty} M_{1} q^{n}\|F\|_{C_{0}^{\beta}(\partial G)} \leq \frac{M_{1} q}{(1-q)} q^{m}\|F\|_{C_{0}^{\beta}(\partial G)^{.}}
\end{gathered}
$$

Easy calculation yields that $T_{h, R} \varphi=F$. So, $u$ is a solution of the problem (2.2) by Section 7 .

According to Lemma 5.1, there is a constant $M_{2}$ such that

$$
\sup _{x \in G}\left|\mathscr{T}_{R} \psi(x)\right|+\sup _{x \in G}\left|\nabla \mathscr{S}_{R} \psi(x)\right| \leq M_{2}\|\psi\|_{C_{0}^{\beta}(\partial G)}
$$

for each $\psi \in C_{0}^{\beta}(\partial G)$. Since $u-u_{m}=\mathscr{Y}_{R}\left(\varphi-\varphi_{m}\right)$, we get from (9.18) and (9.19) that

$$
\sup _{x \in G}\left|u-u_{m}\right|+\sup _{x \in G}\left|\nabla u(x)-\nabla u_{m}(x)\right| \leq M_{2} M_{1} q(1-q)^{-1} q^{m}\|F\|_{C_{0}^{\beta}(\partial G)^{\circ}}
$$

According to Lemma 6.1, there is a constant $M_{3}$ such that

$$
\sup _{x \in G}|\mathscr{\psi} \psi(x)|+\sup _{x \in G}|\nabla \mathscr{D} \psi(x)| \leq M_{3}\|\psi\|_{C_{0}^{1+\beta}(\partial G)}
$$

for each $\psi \in C_{0}^{1+\beta}(\partial G)$. Using (9.17), (9.19), and (9.21), we get

$$
\sup _{x \in G}|u|+\sup _{x \in G}|\nabla u(x)| \leq M_{2} M_{1}(1-q)^{-1}\|F\|_{C_{0}^{\beta}(\partial G)}+M_{3}\|g\|_{C_{0}^{1+\beta}(\partial G)} .
$$

Denote

$$
\begin{gathered}
T \psi(x)=\frac{\partial \mathscr{D} \psi(x)}{\partial n}+h(x) \mathscr{\psi} \psi(x), \quad x \in \partial G \backslash \Gamma, \\
T \psi(x)=h(x)\left(\frac{1}{2} \psi(x)+\frac{1}{2 \pi} \int_{\Gamma} \frac{n^{+}(y) \cdot(y-x)}{|x-y|^{2}} \psi(y) d \mathscr{H}_{1}(y)\right), \quad x \in \Gamma .
\end{gathered}
$$


Then $T$ is a linear operator from $C_{0}^{1+\beta}(\partial G)$ to $C_{0}^{\beta}(\partial G)$ by Section 7. Since

$$
[\mathscr{D} \psi]_{+}(x)=\frac{\psi(x)}{2}+(2 \pi)^{-1} \int_{\Gamma} \psi(y)|x-y|^{-2} n^{+}(y) \cdot(y-x) d \mathscr{H}_{1}(y)
$$

for $x \in \Gamma$ (see [9, Theorem 1]), the operator $T$ is a bounded operator from $C_{0}^{1+\beta}(\partial G)$ to $C^{0}(\partial G)$ by Lemma 6.1. Therefore, $T$ is a closed linear operator from $C_{0}^{1+\beta}(\partial G)$ to $C_{0}^{\beta}(\partial G)$. According to the closed graph theorem (see [13, Chapter II, Section 6, Theorem 1]), there is a constant $M_{3}$ such that $\|T \psi\|_{C_{0}^{\beta}(\partial G)} \leq M_{3}\|\psi\|_{C_{0}^{1+\beta}(\partial G)}$ for each $\psi \in C^{1+\beta_{0}}(\partial G)$. Therefore,

$$
\|F\|\left\|_{C_{0}^{\beta}(\partial G)} \leq\right\| f\left\|_{C_{0}^{\beta}(\partial G)}+M_{3}\right\| g \|_{C_{0}^{1+\beta}(\partial G)} .
$$

We get requested inequalities using (9.18), (9.20), (9.22), and (9.26).

\section{References}

[1] H. Heuser, Funktionalanalysis, B. G. Teubner, Stuttgart, 1975.

[2] C. E. Kenig, Harmonic Analysis Techniques for Second Order Elliptic Boundary Value Problems, CBMS Regional Conference Series in Mathematics, vol. 83, American Mathematical Society, Rhode Island, 1994.

[3] J. Král, Integral Operators in Potential Theory, Lecture Notes in Mathematics, vol. 823, Springer, Berlin, 1980.

[4] P. A. Krutitskii, Explicit solution of the jump problem for the Laplace equation and singularities at the edges, Mathematical Problems in Engineering 7 (2001), no. 1, 1-13.

[5] _ The jump problem for the Laplace equation, Applied Mathematics Letters 14 (2001), no. 3, 353-358.

[6] _ The modified jump problem for the Helmholtz equation, Annali dell'Università di Ferrara. Nuova Serie. Sezione VII. Scienze Matematiche 47 (2001), 285-296.

[7] N. S. Landkof, Fundamentals of Modern Potential Theory, Izdat. "Nauka”, Moscow, 1966.

[8] K. B. Laursen and M. M. Neumann, An Introduction to Local Spectral Theory, London Mathematical Society Monographs. New Series, vol. 20, Oxford University Press, New York, 2000.

[9] V. G. Maz'ya, Boundary integral equations, Analysis, IV, Encyclopaedia of Mathematical Sciences, vol. 27, Springer, Berlin, 1991, pp. 127-222.

[10] D. Medková, The oblique derivative problem for the Laplace equation in a plain domain, Integral Equations and Operator Theory 48 (2004), no. 2, 225-248.

[11] C. Miranda, Partial Differential Equations of Elliptic Type, Ergebnisse der Mathematik und ihrer Grenzgebiete, vol. 2, Springer, New York, 1970.

[12] M. Schechter, Principles of Functional Analysis, Graduate Studies in Mathematics, vol. 36, American Mathematical Society, Rhode Island, 2002.

[13] K. Yosida, Functional Analysis, Berlin, Springer, 1965.

Dagmar Medková: Department of Technical Mathematics, Faculty of Mechanical Engineering, Czech Technical University, Karlovo Nám. 13, 12135 Praha 2, Czech Republic

E-mail address: medkova@math.cas.cz 


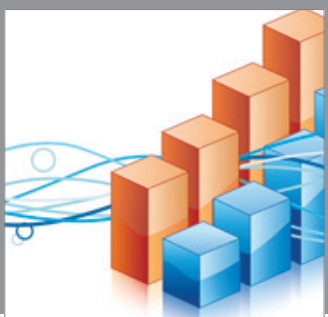

Advances in

Operations Research

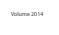

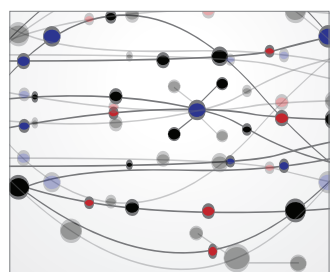

\section{The Scientific} World Journal
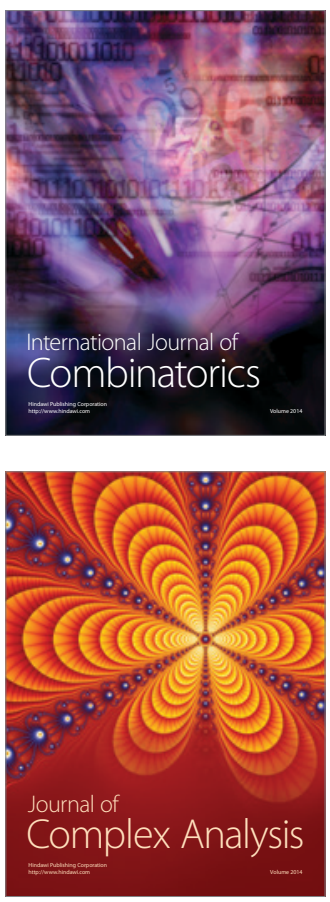

International Journal of

Mathematics and

Mathematical

Sciences
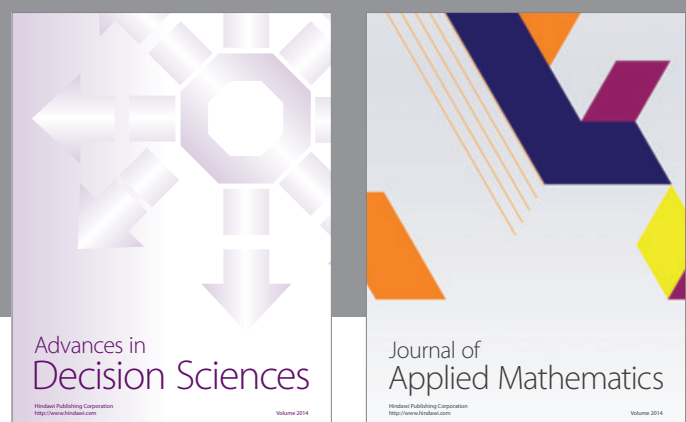

Journal of

Applied Mathematics
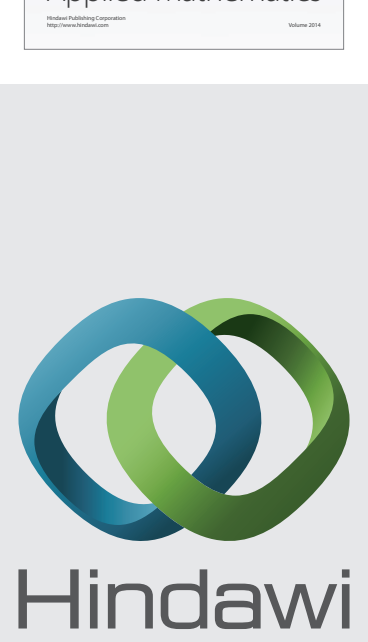

Submit your manuscripts at http://www.hindawi.com
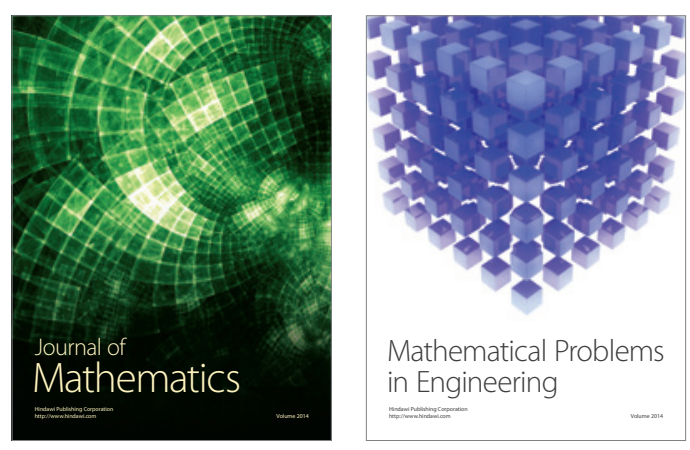

Mathematical Problems in Engineering
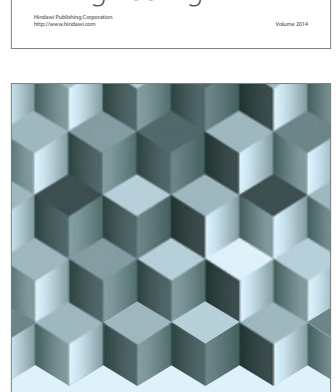

Journal of

Function Spaces
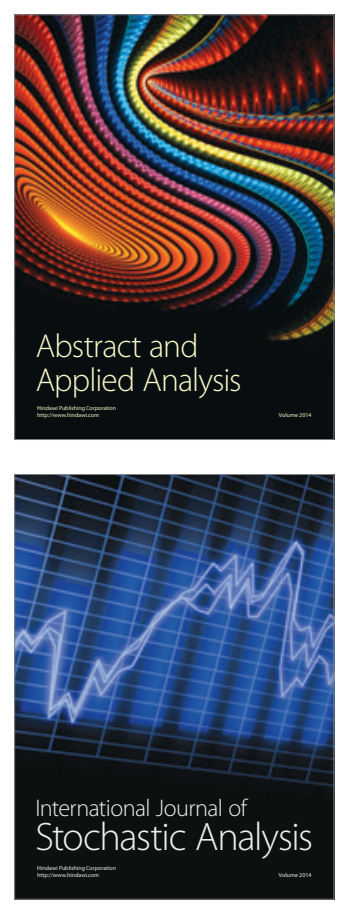

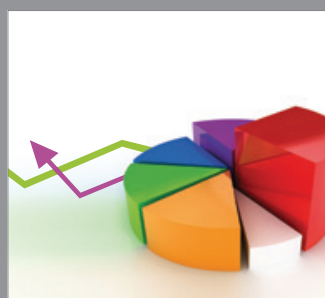

ournal of

Probability and Statistics

Promensencen
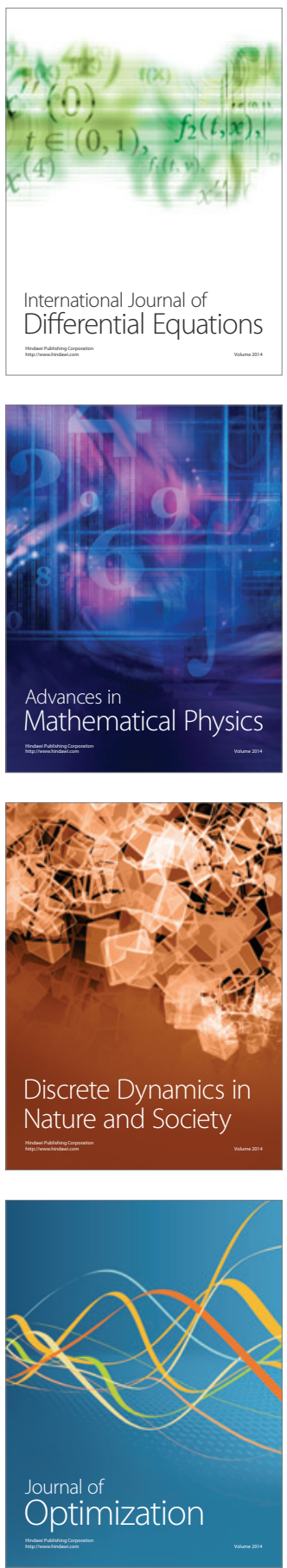